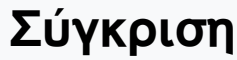

Tóp. 13 (2002)

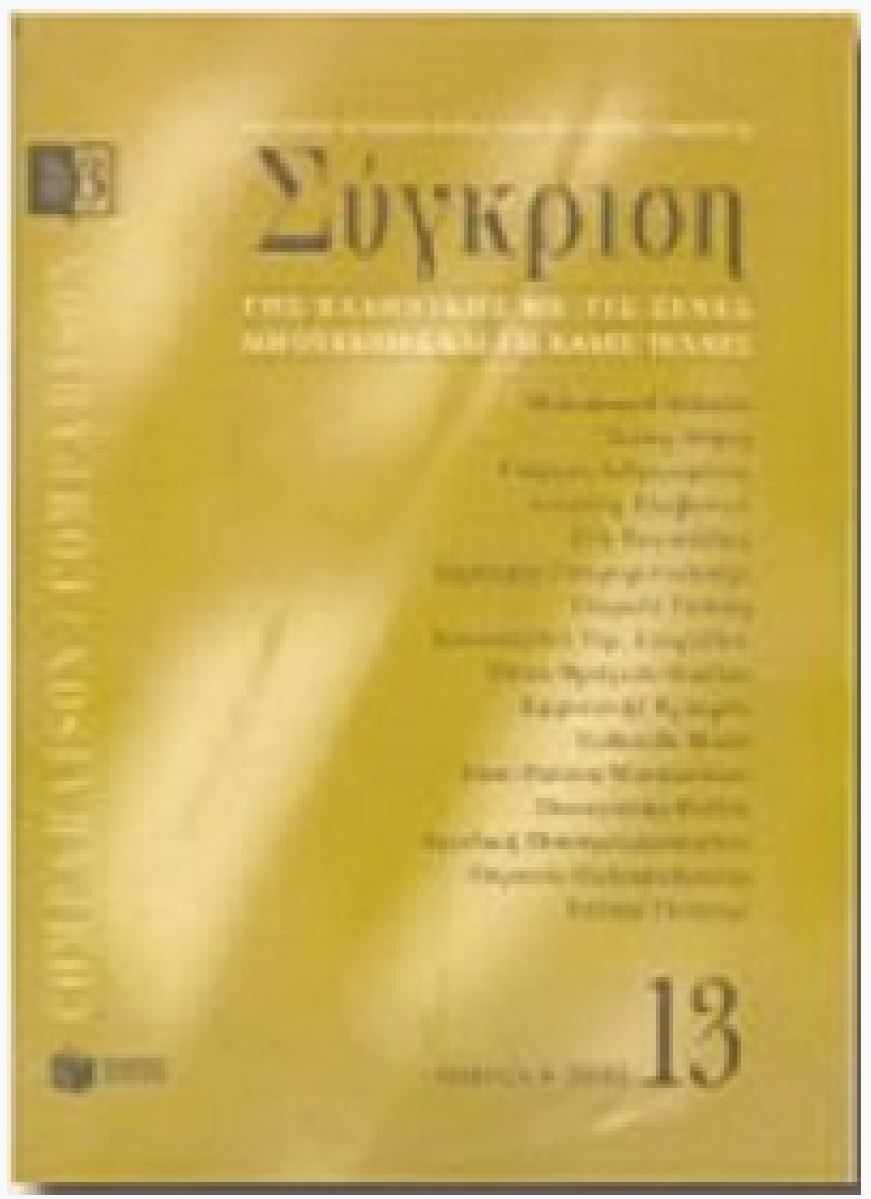

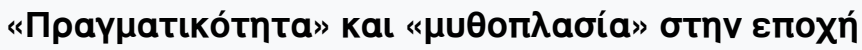

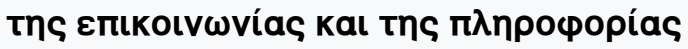

Eleni Politou-Marmarinou

doi: $10.12681 /$ comparison.10139

Copyright $\odot$ 2016, Eleni Politou-Marmarinou

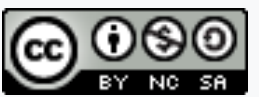

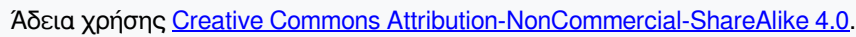

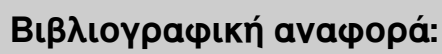

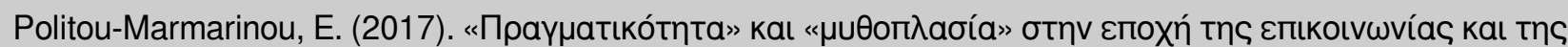

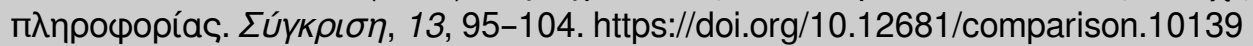




\section{«Réalité» et «fiction» à l'âge de la communication et de l'information*}

1. Le monde créé par la littérature est fictif, dans le sens qu'il ne s'identifie jamais complètement au monde réel. Cette fictionnalité, toutefois, n'empêche pas la distinction des genres littéraires selon leur convergence ou leur divergence par rapport à la réalité. Sur l'axe monde réel $\rightarrow$ non réel, la fiction narrative qui déroule une histoire dans le temps se trouve plus proche du premier pôle, tandis que la poésie lyrique se place plus près du second. En fait, si nous envisageons la relation du monde de la littérature avec le monde réel du point de vue de la temporalité, à laquelle sont inévitablement liées l'existence humaine et l'existence du monde que nous connaissons, nous pouvons admettre que la fiction renvoie à la réalité de l'écoulement $d u$ temps, à une certaine succession dans le temps. Le roman, prenant en considération sérieuse la dimension temporelle, présente un monde en mouvement tout comme le réel. Par contre, la poésie lyrique s'efforce d'échapper à la fatalité de la linéarité temporelle et de devenir un arrêt réfléchi dans le temps. ${ }^{1}$ On peut dire que, comparée à la poésie lyrique, la fiction a un caractère beaucoup plus référentiel et plus représentatif. C'est pourquoi le lecteur s'attend à reconnaître et comprendre le monde produit par la fiction, à travers les mêmes relations spatio-temporelles et causales que celles par lesquelles il perçoit et il interprète la réalité. Et il est vrai que plusieurs des éléments, des modes et des techniques de la fiction ont justement pour but de répondre à cette attente du lecteur. La fiction a toujours eu besoin de la réalité pour se définir. La poésie lyrique, au contraire, plus philosophique que l'histoire, selon Aristote, d'un caractère plutôt autoréférentiel que référentiel, renvoie souvent son lecteur non pas à une réalité liée à l'espace et au temps et donc familière et connue, mais à une réalité «autre», inconnue et unique. ${ }^{2}$

La variabilité de la relation de la fiction avec la réalité n’a pas autant d'importance que la relation elle-même. Car, si la variabilité détermine

* Communication présentée au XVIe Congrès de l'Association Internationale de Littérature Comparée Transitions et transgressions à l'âge du multiculturalisme (Pretoria, 13-19 août 2000). 
chaque fois le mode représentatif et la poétique de la fiction (réaliste, naturaliste, symboliste, surréaliste, structuration cohérente et intelligible, mimétique même, ou bien production antireprésentative et autonome), la relation en soi avec le réel constitue une présupposition pour l'existence de la fiction comme telle. ${ }^{3}$

2. Mais qu'est-ce que la réalité? Ou, au moins, que considérions-nous comme réalité jusqu'à présent? Consultons les dictionnaires.

Au sens courant, réalité est tout ce qui n'est pas invention, illusion ou apparence. Réalité sont les choses elles-mêmes, les faits, la vie, l'existence, opposés à l'imagination ou à la représentation de ce qui existe. En philosophie -mettant à part Platon qui considérait comme réel le monde des idées, dont le monde matériel n'est qu'un pâle refletla réalité c'est le caractère de ce qui consiste en une chose ou concerne une chose. Réel est ce qui existe actuellement et concrètement, qui agit et qui produit des effets, opposé à ce qui est possible, abstrait ou simplement intelligible.

La réalité présentée à l'esprit humain fait l'objet de la connaissance. Réel est ce qui, tangible et visible, est vu, vécu, perçu par un sujet connaissant. Ce sujet complète son expérience de la réalité par les connaissances et les descriptions du réel que lui offre la science. Ainsi la réalité, vue, vécue et connue par un sujet qui profite en même temps des notions indubitables de la science, devient synonyme du vrai, de l'authentique, et s'oppose à l'imaginaire, au fictif, au virtuel.

3. Vers la fin des années 1970 la pensée postmoderne, considérant le discours plutôt comme constructeur du monde, en tant que simple série de faits narratifs, que comme représentation d'une réalité qui existe en fait, avait déjà mis en question la distinction entre ce qui est vrai et ce qui ne l'est pas. Cette relativisation de la vérité a conduit à des constructions mentales affranchies de toute obligation d'être vérifiées et acceptées ou démenties et rejetées. Ainsi, face à Athina, déesse grecque de la sagesse à laquelle le Parthénon étincelant dans sa blancheur a été dédié, une «black Athene», une «Minerve noire» peut trouver une place égale.

Les nouvelles technologies de la communication et de l'information en insérant la notion de la «réalité virtuelle» ont accéléré et aggravé les choses. La réalité, ayant elle-même devenue problématique, pose une nouvelle problématique sur ses rapports avec la fiction littéraire:

- Quelle est/sera l'attitude de la fiction face à cette nouvelle réalité «virtuelle», qui semble lui usurper son statut ontologique ou limiter l'espace traditionnel de son existence? 
- Quel est/sera le caractère futur de la fiction, où puisera-t-elle sa matière pré-textuelle et sa thématique, quelles sont les voies que nos romanciers vont suivre?

- Par rapport à quelle réalité la fiction littéraire sera-t-elle définie, autant au niveau de son écriture qu'au niveau de sa réception?

C'est ce genre de questions que j'essayerai d'explorer dans ce qui suit.

4. Le développement récent des médias, qui permettent la communication directe et la diffusion massive de l'information, minant toutes sortes de frontières (spatiales, temporelles, culturelles, etc.), a entraîné un élargissement énorme des limites traditionnelles de la réalité ainsi que des limites traditionnelles de la connaissance de cette réalité.

$\mathrm{Si}$ on se concentre sur la présentation de la réalité et sur l'information qui la concerne en commençant par la télévision, on constate que le spectateur, sans se déplacer dans l'espace, peut - ou il lui semble qu'il peut- se trouver à n'importe quel endroit de la planète et à n’importe quel moment. L'immédiateté de l'événement rapporté, accompagnée de la toute-puissance de l'image, crée chez le spectateur l'illusion de participer au devenir mondial, de «vivre» les événements, de les connaître de première main. Il en acquiert une expérience «vécue», égale à celle que lui procure son contact avec la réalité quotidienne. Le «je l'ai vu à la télévision» s'identifie, en tant que processus cognitif, au «je l'ai vu de mes propres yeux». Les images électroniques, revêtues d'une valeur psychologique équivalente à celle d'une sensation réelle, non seulement élargissent l'horizon du réel mais, en plus, elles sont stoquées en mémoire comme des connaissances authentiques, au degré où ce qui est vu s'identifie à ce qui est vrai et donc «croyable». ${ }^{4}$ Mais dans quelle mesure ces images $d u$ monde correspondent-elles au monde existant?

Les journaux télévisés quotidiens visent, bien sûr, à l'information, une information qui se veut globale, objective et authentique. Cependant, comme il est impossible et peut-être inutile de rapporter tout ce qui se passe sur notre planète, pour informer il faut sélectionner. Le problème est que les critères de la sélection de ce qui mérite d'être présenté demeurent obscurs et vivement contestés. Deux exemples flagrants: ${ }^{5}$

- Les gens pauvres, les consommateurs les moins privilégiés, constituent le tiers de la population de la terre. Dans l'image du monde donnée par la télévision ils n’occupent que $1,2 \%$ au lieu de $33 \%$.

- Dans le monde «imaginaire» construit par les images —et les informations, les commentaires, etc. — de la télévision, les jeunes et les personnes âgées sont représentés par le tiers et le cinquième, respectivement, de leur proportion réelle dans la population. 
Mais la sélection de la matière présentée n'est pas la seule à faire problème. Ceux des événements qui sont choisis pour nous informer sur ce qui se déroule sur la scène $d u$ monde, sont en outre projetés à l'aide de la technologie que la mise en scène a à sa disposition: montage, effets lumineux et acoustiques, musique, etc. Il en résulte un artifice, construit selon la perception, l'idéologie, les intentions et même les engagements explicites ou latents d'un sujet, selon le plan d'un sujet. ${ }^{6}$ dont, par ailleurs, on ignore l'identité. On ne peut plus être sûr de la vérité de cette «réalité», de l'exactitude des informations, de la validité des commentaires. En voici deux autres exemples: ${ }^{7}$

-'A quel point l'information sur la guerre du Golfe, malgré l'actualisation énorme de CNN -ou, peut-être, à cause d'elle?- a-t-elle été exacte, quand l'humanité entière était menée à croire que les bombes qui tombaient sur les villes nattaquaient que des cibles militaires, qu'il n'y avait pas de victimes parmi les non-combattants, quand les informations sur les victimes des Américains et les vrais buts de la guerre faisaient défaut tout simplement, ou quand les «informateurs» utilisaient une image «vraie» pour nous dire un mensonge? Limage du cormoran asphyxié dans le mazout du Golfe a fait le tour du monde, elle a «informé» et ému des milliers de spéctateurs. Ce n'est que beaucoup plus tard qu'on a appris que le pauvre oiseau était mort à un autre endroit, pendant une autre catastrophe, à une autre époque....

-La même incertitude informative résulte de l'image télévisuelle de la guerre récente de l'OTAN contre la Yougoslavie. Qui est persuadé que l'une des parties seulement a agi criminellement et que l'autre a agi selon la justice en lançant une guerre qu'elle a baptisée, en un oxymore ridicule, «humanitaire»?

Malgré la capacité de la technologie de reproduire exactement et fidèlement, capacité dans laquelle l'humanité mettait son espoir de connaître plus et mieux la réalité, ce que les médias nous offrent c'est l'image d'un monde artificiel, une image qui avance éventuellement - ou souvent- des vérités inexistantes, des événements falsifiés. Loutil, certes, c'est un miroir, mais son usage le rend déformant.

5. Internet, forme technologique nouvelle de la communication et de l'information, produit un espace virtuel d'existence, qui pose de nouveaux problèmes pour le jugement de ce qui est authentique. Internet fonctionne en relation directe avec les mass media et notamment la télévision, car il construit sur l'objectivation d'une expérience massive qui transcende de loin les frontières nationales. ${ }^{8}$ C'est cette expérience massive qui constitue la base commune, au nom de laquelle les visiteurs 
et les usagers d'Internet peuvent communiquer. De ce point de vue, la réalité virtuelle est doublement fictive.

Sur Internet l'information est fournie surtout par des textes qui, sous forme de contributions aux «newsgroups», nous informent sur ce qui se passe dans le monde, et par la production de pages web. Mais quelle est la validité de l'information communiquée par ces textes? Voilà comment un journaliste évalue les informations qu'Internet met à sa disposition: «Internet est un lieu dangereux pour puiser des informations. Elles voyagent très vite et très largement et chacun dit ce qưil veut sur n'importe quoi et les gens ont la tendance de croire ce qu'ils lisent sans s'intéresser à vérifier la source de l'information. C'est un gigantesque bavardage, une machine à produire des rumeurs $\gg .^{9}$

$\mathrm{Au}$ problème de la mal-information ou de la demi-information de la réalité télévisée s'ajoute, pour la réalité virtuelle d'Internet, celui de la super-information. Les gens, qui, de toute façon, ont une confiance aveugle dans la technologie (qui pense vérifier un calcul fait à la machine?) n’ont pas non seulement le temps mais surtout la possibilité de «vérifier la source des informations» - même s'ils pensent qu'ils doivent le faire. Le sens de la source nous mène devant le problème des identités et leurs interférences dans l'espace cybernétique. ${ }^{10}$

Il est vrai que l'identité comme un rôle joué peut exister dans les relations de la vie sociale, que la plupart des usagers d'Internet ne se construisent probablement pas - au moins consciemment- de nouvelles identités ou que «le jeu des identités» sur Internet n'est qu'une manifestation partielle d'un phénomène culturel contemporain beacoup plus général, qui implique la fragmentation du soi ${ }^{11}$ ou la marginalisation progressive du sujet. Mais il est vrai aussi que l'absence d'interaction face-à-face, l'anonymat visuel, crée pour les usagers d'Internet une situation communicative favorable pour «jouer» avec leurs identités. Profitant de la distance entre le virtuel et le réel, entre ce qui peut se passer «online» et ce qui existe «offline», les gens peuvent beaucoup plus facilement qu'ailleurs exploiter des rôles, revêtir une persona, se donner des identités qu'ils voudraient bien avoir ou qui sont supprimées dans leur vie réelle. ${ }^{12}$

Les pages web ont toutes les caractéristiques d'un texte: selon leurs intentions et les lecteurs imaginaires auxquels elles s'adressent, elles exposent et soulignent l'identité de leurs auteurs ou bien elles la dissimulent et l'éliminent. Dans le premier cas l'authenticité de l'information est fortifiée par la présentation et l'insistance sur l'identité réelle de l'auteur (spécialiste, chercheur, professeur d'Université, etc.), c'est-à-dire par une référence directe à la réalité «offline». Dans le deuxième cas, au contraire, l'authenticité de l'information peut être 
renforcée par l'effacement de l'identité de l'auteur (emploi de la troisième personne ou de la première personne du pluriel, style neutre d'un exposé scientifique, objectif, etc.) ou par appel à des sources d'information respectées et incontestables (Institutions, recherches, etc.).

On doit déjà percevoir les analogies entre la «réalité» télévisuelle ou virtuelle et la fiction littéraire:

- Sélection de la matière, structuration et présentation selon le plan d'un sujet.

- Version, interprétation, explication de la réalité, pareilles à celles que présente à ses lecteurs l'auteur d'un roman.

- Les «auteurs» des textes d'Internet, tout comme l'auteur d'un roman, peuvent jouer des rôles, se cacher derrière des personnages, multiplier leur identité (le Madame Bovary c'est moi de Flaubert).

- L'écriture de la réalité virtuelle a même sa stylistique, tout comme le produit fictif littéraire: selon le point de vue et la voix de celui qui parle, on discerne déjà le style objectif, scientifique et neutre, sous lequel disparaît l'identité de l'auteur —un style donc qui correspond à l'écriture littéraire réaliste, naturaliste etc. - du style subjectif, impressionniste, comparable à l'écriture de mouvements littéraires tels le romantisme, le symbolisme, le surréalisme, etc.

- La réalité virtuelle, enfin, fonctionne, en ce qui concerne sa réception, dans le même double sens que la fiction littéraire: elle est non seulement utile, en tant qu'informative, mais agréable aussi, charmante et absorbante, fonction double qui nous rappelle le «dulce et utile» littéraire du vieil Horace.

Il existe pourtant une grande différence entre le monde virtuel et le monde créé par la littérature: le lecteur d'un roman a la conscience que ce qu'il voit se dérouler devant lui n'est pas le monde tangible mais «le monde» fictif d'un écrivain d'identité connue —ou qui peut être connue qui le construit selon son idéologie, son attitude à l'égard de la vie et de l'homme, ses valeurs etc., tandis que le spectateur d'images ou le lecteur de textes électroniques, à cause de sa confiance dans les technologies, le «vu» et le «vécu», ne prend pas conscience (ou, au moins, pas toujours) de la fictionnalité du monde télévisé ou virtuel et l'identifie facilement et presque automatiquement au réel.

Je vais achever cette présentation sur quelques pensées, concernant le rapport entre réalité médiatisée et fiction, comme une première réponse à la problématique posée au commencement de ma communication. Ces pensées, toutefois, étant peut-être prématurées, risquent d'être jusqu'à un certain point arbitraires.

6. Les nouvelles technologies de l'image, de la communication et de

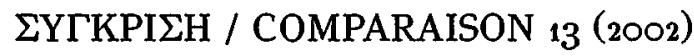


l'information, se servant de leur capacité de reproduction immédiate, fidèle et directe, ont, d'un côté, «agrandi démesurément la réalité aux dimensions du cosmos». ${ }^{13}$ Dans cette nouvelle réalité la sûreté d'une marche linéaire (temporelle, idéologique, culturelle, économique, professionnelle, personnelle, familiale, etc.) peut être interrompue à tout moment par le hasard d'un événement, ${ }^{14}$ inexplicable par la rationnalité de notre culture, dans le cadre d'une société jusqu'aujourd'hui rassurée dans sa foi en un déroulement temporel-causal de l'histoire. La technologie dévoile les aspects insoupçonnés et incroyables de la réalité et rend au monde «son inquiétante étrangeté». ${ }^{15}$ C'est un réel qui dépasse l'imaginaire.

Transportées au niveau littéraire ces expériences expliquent peut-être le succès de la science-fiction ou des romans d'épouvante, à l'intérieur d'un genre fictif qui veut, pourtant, rester représentatif et référentiel. Aujourd'hui, ce genre de fiction littéraire cesse d'être «un jeu de l'esprit», un «exercice mental» et, par conséquent, n' appartient plus à un domaine littéraire inférieur ou marginal. Il manifeste quelque chose de plus profond. C'est un genre qui «affiche dans un univers romanesque inquiétant l'irréalité d'un univers qui ne croit plus lui-même à ses attaches avec le réel».16

En même temps, la rapidité et la massivité de la communication et de l'information, qui transcendent les limites et abolissent toute sorte de frontières, ont amené une homogénéisation tant de la réalité représentée, où les différences (culturelles et autres), les altérités, les minorités, sont atténuées ou littéralement effacées, que de la réception et de l'experience massive de cette réalité. Il semble qu'à une première étape, la fiction n'a pas pu échapper au piège de la représentation réaliste de cette réalité globalisée et nivelée. Adoptant les conventions du modèle communicatif souverain et le «réalisme» des medias, elle s'est vouée à la production massive de romans qui peuvent très bien se situer à nimporte quel endroit du globe, dont les héros mènent une vie similaire. Ce sont des oeuvres qui, de décodage facile, sont agréablement accessibles au grand public, auprès duquel elles trouvent souvent un grand succès. Mais, comme la critique littéraire l'a déjà remarqué pour la fiction grecque contemporaine, la question posée par ces oeuvres est de savoir si, avec elles, les possibilités de la fiction «se limitent à décorer simplement ce qui est évident $»{ }^{17}$

D'un autre côté, la manipulation de la réalité présentée par les technologies, son simulacre virtuel, fournissent à l'homme contemporain, au lieu d'une connaissance authentique, l'expérience d'une illusion. On peut espérer, pourtant, que les écrivains, sujets créateurs parce que plus sensibles et plus susceptibles que l'homme commun de comprendre ce 
qui se passe autour d'eux, seront de plus en plus conscients de la distance entre la réalité existante et la réalité médiatisée. Il me semble qu'alors la fiction littéraire n’aura que deux chemins possibles à suivre. Si elle veut garder son caractère générique représentatif, elle dénoncera, probablement, cette discordance par le biais éventuel de la parodie et de l'ironie. Si, en outre, la fiction doit dans l'avenir tourner le dos à cette réalité trompeuse, abandonnant la représentation et la référence, il est possible qu'elle devienne de plus en plus autoréférentielle et autoréflexive. Dans ce cas, la plongée dans les profondeurs psychiques de l'inconscient et la magie des images émergées remplaceront la référence à une réalité inévitablement confondue avec ses simulacres. ${ }^{18}$ Lécriture romanesque pourrait alors rendre explicite la rupture entre les mots et les choses. Elargissant et approfondissant la dichotomie entre réalité et langage la fiction future pourrait ainsi devenir de plus en plus «poétique».

\section{Notes}

${ }^{1}$ Cf. René Wellek and Austin Warren, Theory of Literature, Penguin Books, [1970], p. 215.

${ }^{2}$ Cf. Kate Hamburger, Logique des genres littéraires. Traduit de l'allemand par Pierre Cadiot. Préface de Gérard Genette. Seuil, Paris 1986. pp. 248-250.

${ }^{3}$ Jean Bessière dans son texte «Littérature et représentation» (Théorie littéraire. Publié sous la direction de Marc Angenot, Jean Bessière, Douwe Fokkema, Eva Kushner. PUF, Paris 1989, pp. 309-324) remarque que «l'autonomie du littéraire nimplique pas le défaut de relation d'objet», et cette remarque, dans la perspective sous laquelle j'envisage les choses ici, me semble plus importante que la caracrérisation de cette relation comme «déplacée et médiée» ( $p$. 312).

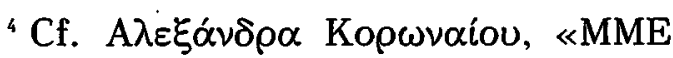

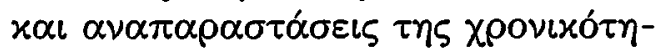

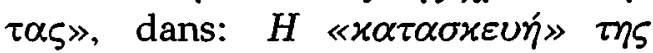

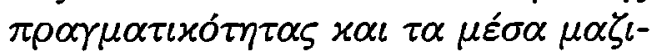

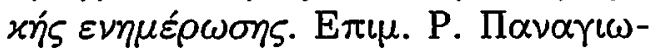

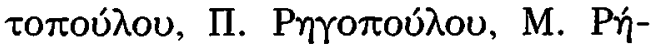

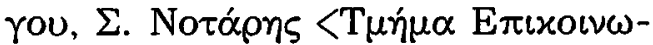

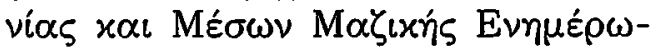
$\sigma \eta \zeta\rangle, \quad \varepsilon x \delta$. A 1998, $\sigma \varepsilon \lambda$. 79-80. Charis Kambouridis constate aussi du point de vue de la

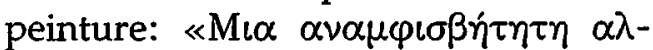

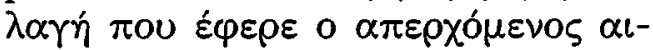

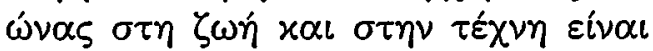

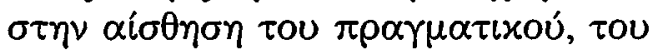
$\beta \iota \omega \mu \varepsilon \dot{v o v, ~ \tau o v ~} \alpha \lambda \eta \theta \iota v o u ́$. H $\varphi \omega \tau 0-$

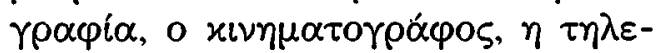

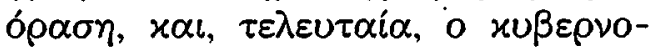

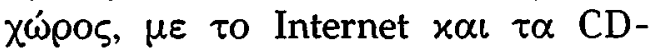
ROM, $\alpha \mu \varphi \imath \sigma \beta \dot{\eta} \tau \eta \sigma \alpha \nu \varepsilon \dot{\varepsilon} \mu \rho \rho \alpha \tau \alpha \tau \eta \nu$

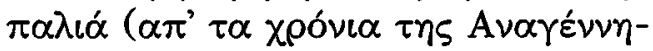

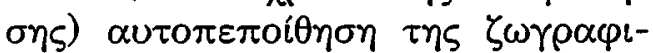




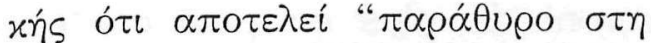

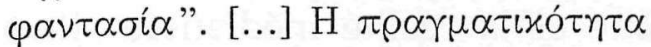

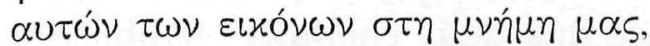

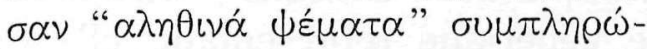

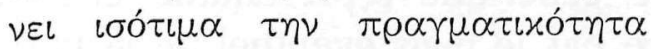
$\tau \omega \nu \quad \alpha \iota \sigma \theta \dot{\eta} \sigma \varepsilon \omega \nu \gg . \quad(« \mathrm{M} \eta \chi \alpha \nu \iota x \dot{\eta} \quad \varphi \alpha-$

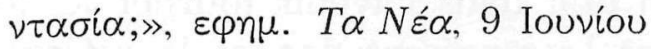

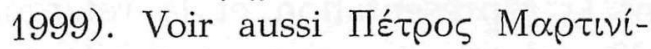

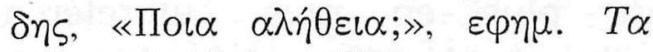

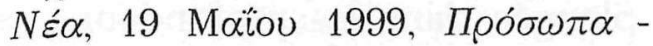
21 o丂 $\alpha \iota \omega \nu \alpha \varsigma, \sigma \varepsilon \lambda .6$.

${ }^{5} \mathrm{Je}$ les puise chez George Gerbner,

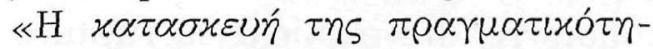

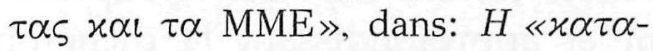

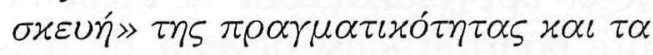

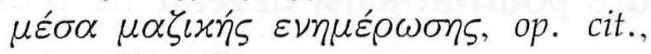

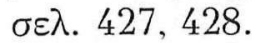

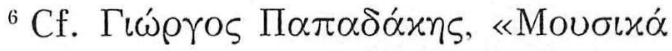
$\delta \rho \alpha ́ \mu \alpha \tau \alpha \quad \sigma \tau \alpha \quad \delta \varepsilon \lambda \tau i \alpha \quad \varepsilon i \delta \dot{\eta} \sigma \varepsilon \omega \nu \gg$,

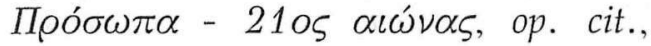
$\sigma \varepsilon \lambda .7$.

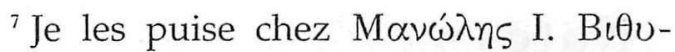

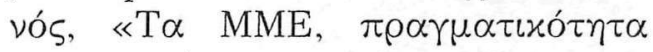

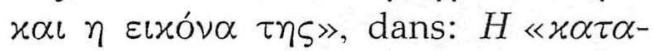

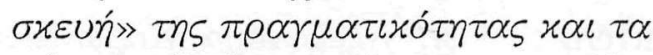

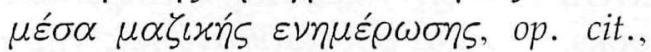

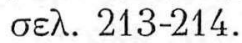

${ }^{8}$ Cf. P. Scannell, Radio, Television and Modern Life, Blackwell, Oxford 1996.

${ }^{9}$ Christine Hine, Virtual Ethnography, Sage Publications, London 2000, p. 123.

${ }^{10}$ Voir, sur ce sujet, le chapitre «Authenticity and Identity in Internet Contexts» de Christine Hine, Virtual Ethnography, op. cit., pp.118-146.

"Cf. Douwe Fokkema, «The Critical Discourse of Identity and its

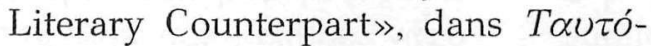

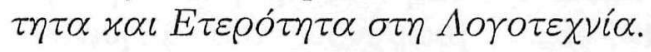

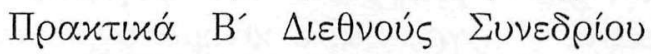

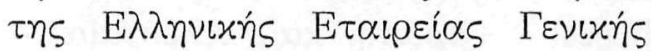

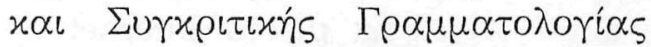

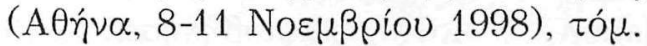

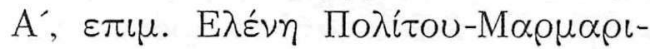

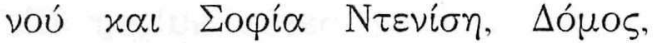

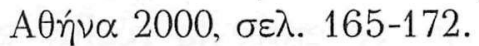

${ }^{12}$ Voir S. Turkle, «Parallel lives: working on identity in virtual space», in Constructing the Self in a Mediated World, D. Grodin and T. R. Lindlof (eds.) Sage Publications, Thousand Oaks, CA, 1996, pp. 156175.

${ }^{13}$ Jeanne-Marie Clerc, «Les technologies de l'image et la transformation d'une culture», dans Précis de Littérature Com parée. Sous la direction de Pierre. Brunel et d'Yves Chevrel, PUF , Paris 1989, p. 295.

${ }^{14} \mathrm{Cf}$. $\mathrm{A} \lambda \varepsilon \xi \alpha \dot{\alpha} \delta \rho \alpha$ Ko $\omega \nu \alpha i o v, ~ « \mathrm{MME}$

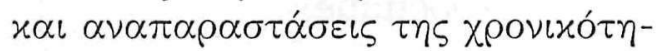
$\tau \alpha \varsigma »$, op. cit., $\sigma \varepsilon \lambda .80-81$.

${ }^{15}$ Jeanne-Marie Clerc, «Les technologies de l'image et la transformation d'une culture», op. cit., p. 293.

${ }^{16}$ Jeanne-Marie Clerc, «Les technologies de l'image et la transformation d'une culture», op. cit., p. 295.

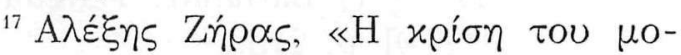

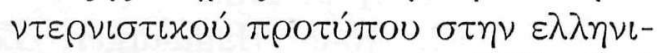

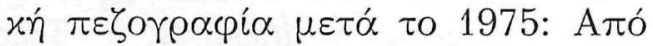

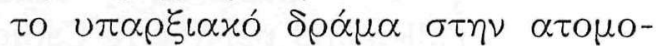

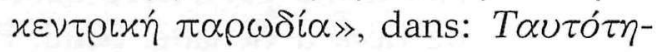

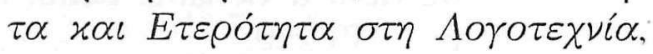
op. cit., $\sigma \varepsilon \lambda .81$.

${ }^{18} \mathrm{La}$ peinture, art visuel par

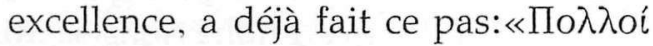

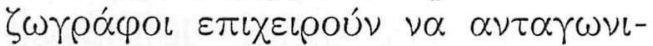

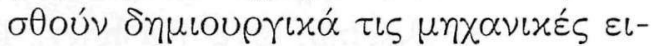

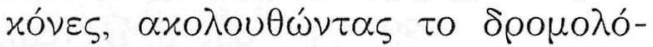

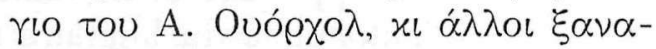

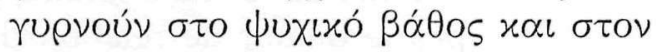
$\alpha \rho \chi \varepsilon ́ \tau U \pi 0 \mu \dot{\theta} \theta 0, \alpha \nu \alpha \zeta \eta \tau \omega \dot{\nu} \tau \alpha \varsigma \mu \alpha \gamma l-$

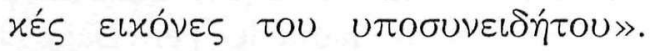

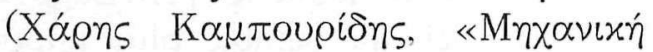

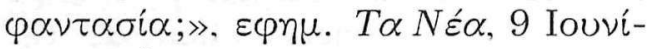
ov 1999). 


\section{$\Pi \varepsilon \rho i \lambda \eta \psi \eta$}

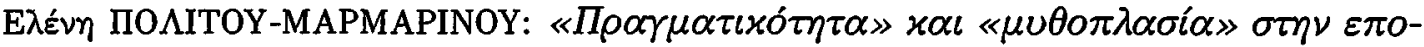

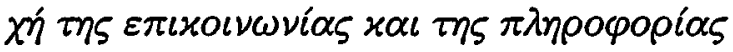

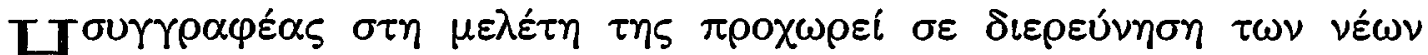

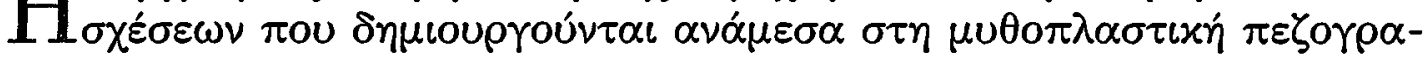

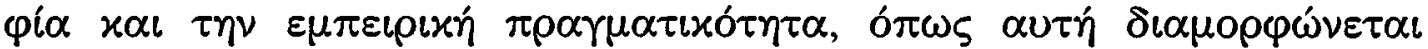

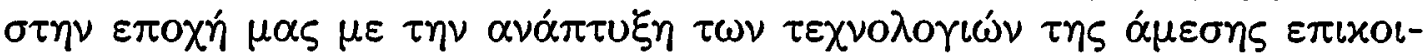

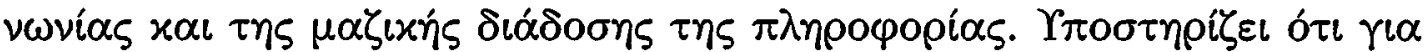

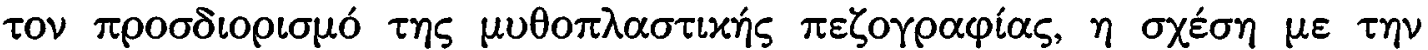

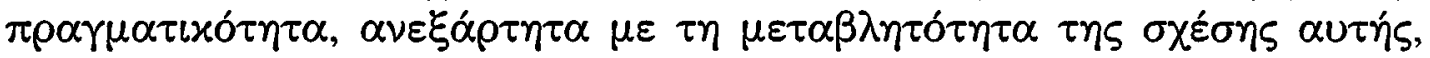

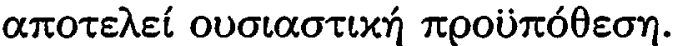

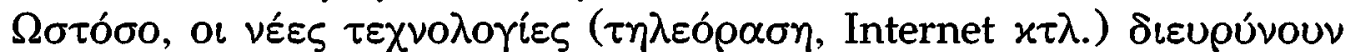

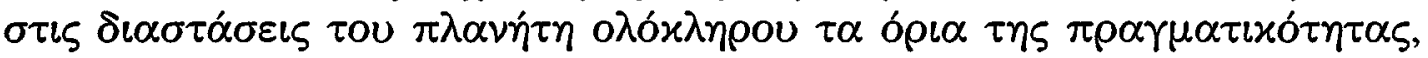

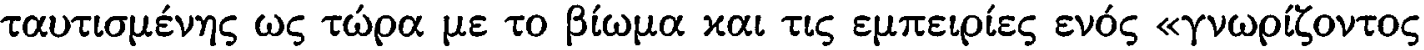

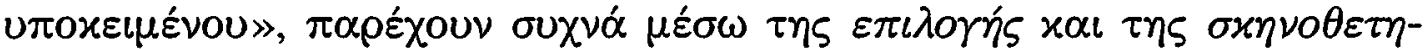

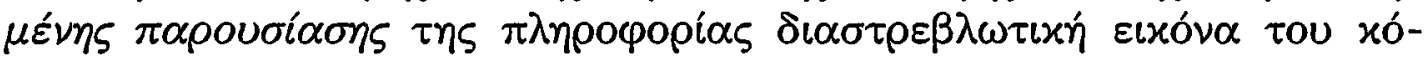

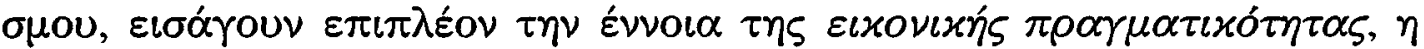

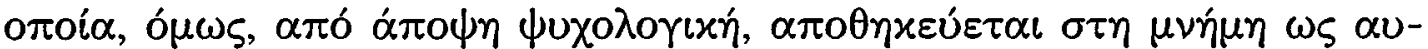

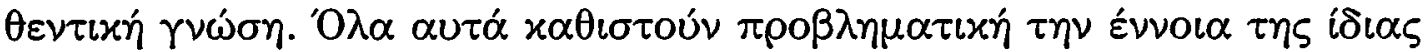

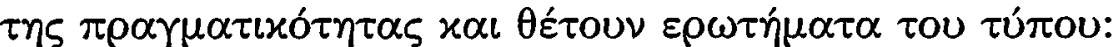

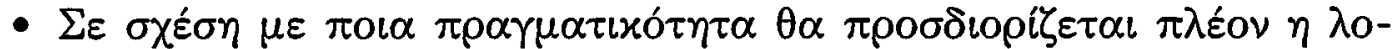

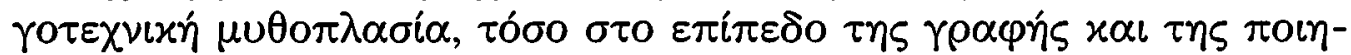

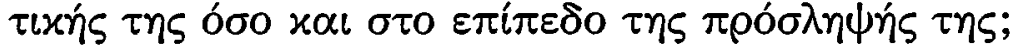

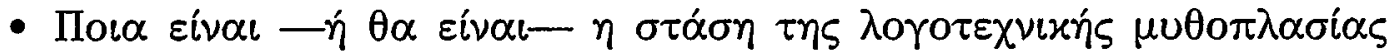

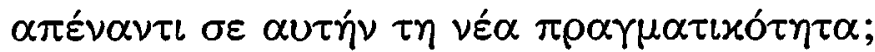

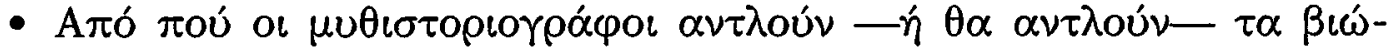

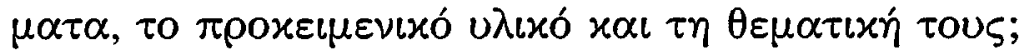

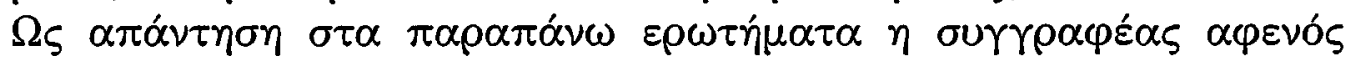

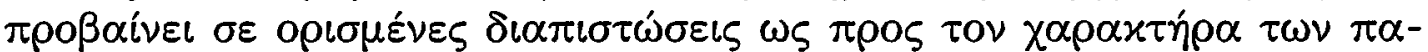

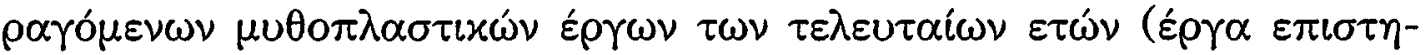

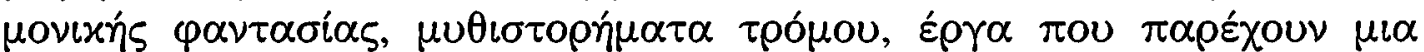

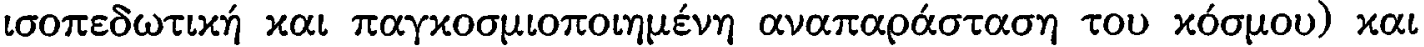

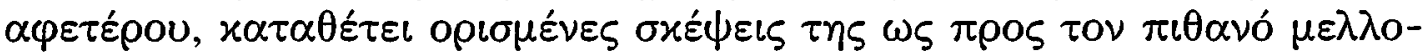

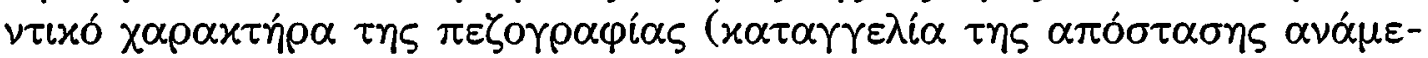

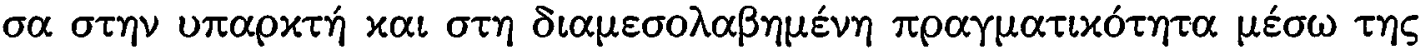

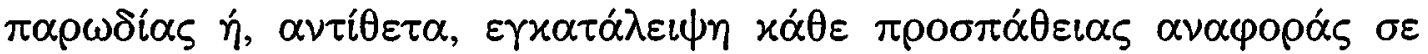

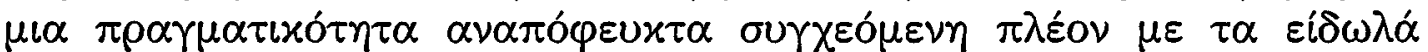

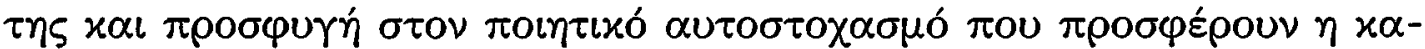

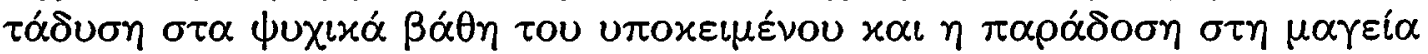

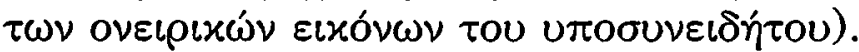

